\title{
Sudden Breakdown in Linear Response of a Rotationally Driven Magnetic Microparticle and Application to Physical and Chemical Microsensing ${ }^{\dagger}$
}

\author{
Brandon H. McNaughton, ${ }^{\ddagger}$, Karen A. Kehbein, ${ }^{\S}$ Jeffrey N. Anker, ${ }^{\ddagger}$, and Raoul Kopelman $*, \hbar, \S$ \\ Applied Physics Program, 2477 Randall Laboratory, The University of Michigan, \\ Ann Arbor, Michigan 48109-1120, and Department of Chemistry, 930 North University, \\ The University of Michigan, Ann Arbor, Michigan 48109-1055
}

Received: January 9, 2006; In Final Form: April 13, 2006

\begin{abstract}
In this work, sensing magnetic microparticles were used to probe both the local $\mathrm{pH}$ and the viscosity-dependent nonlinear rotational behavior of the particles. The latter resulted from a critical transition marking a driven particle's crossover from phase-locking to phase-slipping with an externally rotating magnetic field, i.e., a sudden breakdown in its linear response that can be used to measure a variety of physical quantities. The transition from simple rotation to wobbling is described both theoretically and experimentally. The ability to measure both chemical and physical properties of a system could enable simultaneous monitoring of chemical and physical interactions in biological or other complex fluid microsystems.
\end{abstract}

\section{Introduction}

Taking measurements on smaller and smaller size scales has driven the innovation of new technologies and led to the discovery of entirely new scientific disciplines. While many micro- and nanoprobes have been developed to monitor either physical properties (such as fluid viscosity) or chemical concentrations (such as $\mathrm{pH}$ ), we demonstrate a probe that is capable of probing both on the microscale. In our lab, the drive toward miniaturization of chemical sensors led to the development of photonic explorers for bioanalysis in biologically localized embedding (PEBBLEs). PEBBLEs are microscopic sensors, typically $20-1000 \mathrm{~nm}$ in diameter, composed of a semipermeable polymer or silica matrix containing fluorescent dyes that report local chemical analyte concentrations inside live cells. ${ }^{1-5}$ PEBBLEs occupy a niche that lies between pulled optical fiber optodes and free molecular indicator dyes. PEBBLEs retain the complexity and biocompatibility of optodes, while the size reduction allows delivery of multiple sensors into cells and enables chemical imaging. The pores in the material matrix of the PEBBLEs are designed to allow small chemical analytes to diffuse through the matrix and interact with the dye but prevent direct interaction between the dyes and the protein environment. Thus, encapsulation protects the dye and other components from fluorescence quenching or enhancement and from indicator sequestration or degradation due to cellular components, such as proteins or enzymes. The encapsulation also protects the local environment from interactions with the dye, which allows for toxic dyes to be used. Another benefit is that PEBBLEs offer a platform into which multiple components can be added, allowing design of complex synergistic sensing mechanisms. ${ }^{1-5}$ One useful way the PEBBLE platform can be modified is by the addition of a magnetic material. ${ }^{6,7}$ Once modified with a magnetic material, PEBBLEs can be magneti-

\footnotetext{
† Part of the special issue "Robert J. Silbey Festschrift".

* Author to whom correspondence should be addressed. E-mail: kopelman@umich.edu.

$\doteqdot$ Applied Physics Program.

$\S$ Department of Chemistry.
}

cally manipulated and ultimately used as a chemical sensor that has physical probing capabilities.

While chemical concentrations can be measured through the fluorescence emission of indicator dyes, physical properties have been probed through the rotational behavior of micro- and nanoparticles. ${ }^{8-11}$ For example, when a rigid ferromagnetic particle is rotated at low speeds by a continuously rotating external magnetic field, the particle is phase-locked and, therefore, synchronous with the external field. At higher external rotation rates, the particle cannot keep up with the external field, and there is a sudden breakdown of its linear response. The general property of sudden breakdown in linear-responsive systems even appears to be valid down to single diatomic molecule rotation in liquids. ${ }^{12}$ For particles studied in this paper, the sudden breakdown occurs when the rotation of a particle is no longer phase-locked, becoming asynchronous with the external magnetic field (phase-slipping). In the latter case, the particle rocks back and forth but has a net average rotation rate that is in the same direction as the external rotating field. This average rate actually decreases with increasing rotation rates of the external driving field. The transition from phase-locked to phase-slipping is marked by a critical external rotation rate, where the particle's rotation behavior changes from synchronous to asynchronous (linear to nonlinear).

This "critical slipping onset" is highly sensitive to local physical properties of the environment, and the general type of critical phase-slipping behavior has been previously demonstrated, under varying designations, with a pair of magnetic holes, ${ }^{13}$ optically torqued glass nanorods, ${ }^{9}$ paramagnetic chains, ${ }^{10}$ carbon nanotubes loaded with magnetic nanoparticles, ${ }^{11}$ and magnetic microdrills. ${ }^{14}$ Helgesen and co-workers ${ }^{13}$ investigated this type of nonlinear phenomena in systems with magnetic holes. Shelton and colleagues rotated a glass nanorod under optical rotation and characterized the rotational behavior of single rods. Biswal and Gast ${ }^{10}$ studied the nonlinear behavior in magnetic chains that were used as micromixers. Korneva et al. ${ }^{11}$ examined the rotational behavior of carbon nanotubes loaded with magnetic particles to approximate the total magnetic moment of the loaded tubes. In our lab we have investigated 


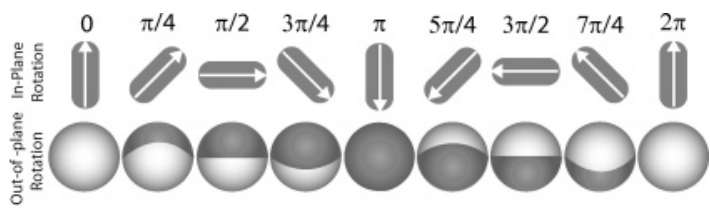

Figure 1. Schematic of phase-locked rotation, below the critical slipping rate, for two types of particles, with each type rotated in a different plane: (top) an asymmetric particle being rotated in the image plane; (bottom) a physically symmetric, but optically asymmetric, magnetically modulated optical nanoprobe (MagMOON), being rotated in a plane orthogonal to the image plane. In both cases, the orientation and average rotation rate will be identical, allowing either technique to be used for phase-locking and phase-slipping experiments.

this critical phase-slipping behavior using $\sim 4.4 \mu \mathrm{m}$ spherical fluorescent magnetic particles. ${ }^{15}$ Through the combination of this critical phase-slipping behavior of a single magnetic particle with chemical sensing, it is possible to measure chemical changes while monitoring physical properties, yielding multiplesensing capabilities.

There are two planes in which micro- and nanoparticles used in critical phase-slipping experiments can be rotated, namely, parallel and perpendicular to the imaging plane. To track the particle rotation and determine whether the particles are phaselocking or phase-slipping, one can rotate an asymmetrical particle in the plane of the sample $\mathrm{e}^{9-11}$-see top of Figure 1. Image analysis software can then be used to track the orientation and, therefore, the average rotation rate. This is the technique used in this paper. An alternative technique ${ }^{15}$ is to rotate magnetically modulated optical nanoprobes (MagMOONs) in a plane that is orthogonal to the imaging plane-see bottom of Figure 1. MagMOONs are half-coated fluorescent magnetic particles that emit varying intensities of light, depending on their angular orientation. ${ }^{6,7,15-22}$ By tracking the intensity of the MagMOON as it rotates, the orientation and average rotation rate can be determined, and therefore, phase-locking and phaseslipping can be monitored for spherical particles. ${ }^{15}$

One potential application for combined physical and chemical sensing probes is for the study of an environment in which a chemical change induces a physical change or vice versa. An example for this can be found in giant unilamellar vesicles, where introduction of a cross-linking ion causes the formation of a hydrogel. ${ }^{23}$ Alternatively, a sensing magnetic particle could be used to apply physical forces to cells and to monitor the chemical and physical responses, such as ion currents in stressactivated ion channels, downstream biochemical responses to changes in stiffness and ion currents, and physical stiffening of the cytoskeleton in response to magnetic forces. ${ }^{24-26}$ In such systems, a physical and chemical probe could be used to reveal new information about the relationship between physical and chemical properties.

In this paper we demonstrate the combination of two types of probes: one that can be used to measure changes in $\mathrm{pH}$ and the other that has a critical phase-slipping behavior that is dependent on physically interesting parameters such as viscosity, temperature changes, single-particle magnetic moment, external magnetic fields and even volume changes due to a biological antigen attaching to the probe. The chemical signal is reported by the fluorescence spectrum of the probes, while the rotational dynamics are reported through a particle's average rotation rate, which is monitored optically as well. In the future, this combination could be used to simultaneously measure physical and chemical values in a biologically interesting environment, such as inside an embryo or cellular cytoplasm.

\section{Theory}

Equation of Motion for a Magnetically Driven Rotor with Drag. The equation of motion for a magnetic particle, in a viscous solution, torqued by an external magnetic field, $B$, is given by ${ }^{27}$

$$
I \frac{\mathrm{d}^{2} \theta}{\mathrm{d} t^{2}}-\gamma \frac{\mathrm{d} \theta}{\mathrm{d} t}=m B \sin (\phi)
$$

where $I$ is the moment of inertia, $t$ is time, $\gamma$ is the drag coefficient, $\theta$ is the angular orientation of the magnetic moment, $m$ is the total magnetic moment of the particle, and $\phi$ is the phase lag, which is the angle between the external magnetic field and the magnetic moment. For the simple case of an external magnetic field rotating continuously in a plane, the magnetic torque is given by $\Gamma_{m}=\mathbf{m} \times \mathbf{B}=m B \sin (\phi)=m B$ $\sin (\Omega t-\theta)$, where $\Omega$ is the rotation rate of the external field. For low Reynolds numbers the inertial term may be neglected. ${ }^{28}$ Thus, rewriting eq 1 in a nondimensional form, after neglecting inertia, reduces the equation of motion to the nonuniform oscillator equation

$$
\frac{\mathrm{d} \phi}{\mathrm{d} \tau}=\frac{\Omega}{\Omega_{\mathrm{c}}}-\sin (\phi)
$$

where $\Omega_{\mathrm{c}}=m B / \gamma, \tau=\Omega_{\mathrm{c}} t$, and $\phi=\Omega t-\theta$. Analogous "nonuniform oscillator" equations have successfully described a variety of systems, including the optical rotation of glass nanorods, ${ }^{9}$ the flashing of fireflies,${ }^{29}$ and the voltage oscillations in Josephson junctions. ${ }^{29}$

Nonuniform Oscillator. Equation 2 is formally known as the nonuniform oscillator equation ${ }^{29}$ and has two solutions that indicate two different types of rotational behavior. If the external magnetic field frequency is below a critical rotation rate, $\Omega_{\mathrm{c}}$, then the magnetic particle will rotate at the same rate as the external field with a phase lag that increases with the external rotation rate, $\phi=\sin ^{-1}(\gamma \Omega / m B)$. The maximum rotation rate occurs when $\phi=\pi / 2$ and $\Omega=\Omega_{\mathrm{c}}$. However, if the external magnetic field frequency is higher than $\Omega_{\mathrm{c}}$, then the magnetic particle experiences a fast rocking motion superimposed on a slower net rotation rate that decreases with increasing external driving rate, exhibiting a nonlinear relationship. The magnetic particle is acting as a nonuniform oscillator with a phase lag that "critically slips" at $\Omega_{\mathrm{c}}$ and can be described, for the simple case of $\gamma=\kappa \eta V$, giving

$$
\Omega_{\mathrm{c}}=\frac{m B}{\gamma}=\frac{m B}{\kappa \eta V}
$$

where $\kappa$ is the shape factor, which for a sphere is $6, \eta$ is the dynamic viscosity, and $V$ is the particle volume. Here we only consider the simple case of a field rotating a particle, in two dimensions, with a resultant constant drag. More complicated critical phase-slipping behavior can result from a variety of scenarios, such as using external magnetic fields strong enough to affect the magnetic moment of the particles or having nonuniformities in the external magnetic field. Similar cases of critical phase-slipping behavior have also been observed in optically torqued glass nanorods, ${ }^{9}$ paramagnetic chains used as micromixers, ${ }^{10}$ carbon nanotubes loaded with magnetic nanoparticles, ${ }^{11}$ and MagMOONs. ${ }^{15}$ The above parameters in eq 3 can be exploited for a variety of applications-see Table 1. For instance, with a given particle and a given field, eq 3 will give the change in viscosity that occurs due to a change in temperature or due to a biological change. 
TABLE 1: Applications of Measurement of the Critical Slipping Rate, $\Omega_{\mathrm{c}}=m B / \kappa \eta V^{a}$

\begin{tabular}{lll}
\hline \multicolumn{1}{c}{ constant } & \multicolumn{1}{c}{ determinable value } & \multicolumn{1}{c}{ applications } \\
\hline$B, \kappa, \eta$, and $V$ & $m$, magnetic moment & measurement of moment, magnetic content, and particle uniformity of synthesized particles. \\
$m, \kappa, \eta$, and $V$ & $B$, magnetic field & measurement of local rotating magnetic fields. \\
$n, \mathrm{~B}, \kappa$, and $V$ & $\eta$, viscosity & measurement of effective viscosity near an interface, local viscosity, and spatial viscosity. \\
$m, B$, and $\eta$ & $\kappa V$, shape factor and volume & detection of single-particle binding events that will result from shape and volume changes.
\end{tabular}

${ }^{a}$ Various physical parameters can be determined, using eq 3 , if a value for the critical slipping rate, $\Omega_{\mathrm{c}}$, is experimentally measured. Also shown are the applications of determining these parameters.

From Linear to Nonlinear: Behavior of Driven and Dragged Rotation. The linear to nonlinear behavior, characteristic below and above the critical slipping rate, can be symbolically described by calculating the average rotation rate of the rotating particle and is given by

$$
\left\langle\frac{\mathrm{d} \theta}{\mathrm{d} t}\right\rangle= \begin{cases}\Omega & \Omega<\Omega_{\mathrm{c}} \\ \Omega-\sqrt{\Omega^{2}-\Omega_{\mathrm{c}}^{2}} & \Omega>\Omega_{\mathrm{c}}\end{cases}
$$

Experimentally, $\langle\mathrm{d} \theta / \mathrm{d} t\rangle$ can be determined from tracking the particle orientation in time and by taking a fast Fourier transform (see Analysis in the Experimental Section). A value for $\Omega_{\mathrm{c}}$ can then be determined by performing a least-squares fit on $\langle\mathrm{d} \theta / \mathrm{d} t\rangle$ versus $\Omega$ (see Results and Discussion).

As can be seen from eq 3 and Table 1 , measurements of $\Omega_{\mathrm{c}}$ allow for the determination of a variety of physical values. Alternatively, if one of the parameters such as viscosity is altered, then the critical slipping rate, $\Omega_{\mathrm{c}}$, will also change. Indeed, this would be the case for varying the temperature in a glycerol environment, ${ }^{30}$ and therefore, the probe could be used as a temperature/viscosity probe. Another parameter that can be manipulated is the particle volume. This can be done by modifying the surface chemistry of the particle so that selectable biological antigens will attach to its surface. For instance, when an antigen is attached, the effective hydrodynamic volume of the magnetic particle will be changed, causing a change in the critical slipping rate. Through the combination of this type of critical phase-slipping behavior with chemical sensing, chemical concentrations could be measured while physical changes are monitored, giving a multiple-sensing platform.

\section{Experimental Section}

Preparation of Sol-Gel Particles with SNARF-1 and Barium Ferrite. From a modification of the Stöber method ${ }^{31}$ developed by Nozawa et al., ${ }^{32}$ approximately $25 \mathrm{mg}$ of 40-60 $\mathrm{nm}$ barium ferrite nanopowder (Sigma-Aldrich) was sonicated for several minutes in $12.5 \mathrm{~mL}$ of ethanol (Sigma-Aldrich), resulting in a weight percent of solids of $0.2 \%$. To this, 2.38 $\mathrm{mL}$ of ammonium hydroxide (Sigma-Aldrich) and $400 \mu \mathrm{L}$ of 5 $\mathrm{mg} / \mathrm{mL}$ SNARF-dextran (Invitrogen/Molecular Probes) were added. This solution was placed in a three-necked roundbottomed flask and purged with argon. A condenser was placed in one of the necks of the round-bottomed flask to keep the reactants from evaporating, and the other two necks were sealed with rubber stoppers. Then, a solution of $7.5 \mathrm{~mL}$ of ethanol and $1.25 \mathrm{~mL}$ of tetraethyl orthosilicate (TEOS) (Sigma-Aldrich) was placed in a $10 \mathrm{~mL}$ syringe. PEEK tubing, with an interior diameter of $0.13 \mathrm{~mm}$ was attached to the end of the syringe and inserted into a rubber-capped neck of the round-bottomed flask so that the end of the tubing was just above the solution level. The syringe was placed in a syringe pump, and the contents were added to the round-bottomed flask at a flow rate of $\sim 0.5 \mathrm{~mL} / \mathrm{h}$. Throughout the addition, the solution in the round-bottomed flask was stirred at a constant rate of $500 \mathrm{rpm}$ and temperature of $20.5^{\circ} \mathrm{C}$. After the complete addition of the solution in the syringe, the reaction was allowed to stir for 12 $\mathrm{h}$. The particles were then rinsed with ethanol via centrifugation using a speed of $5000 \mathrm{rpm}$ for $15 \mathrm{~min}$, and they were collected by filtering through a $0.2 \mu \mathrm{m}$ Whatman Anodisc filter membrane with ethanol.

Viscous pH Buffer Preparation and Suspension. pH buffers (50 mM phosphate) of $\mathrm{pH} 6.6$ and $\mathrm{pH} 8.8$ were prepared and mixed $1: 1$ by mass with $99.5+\%$ glycerol (Sigma-Aldrich). This produced a 0.5 glycerol mass fraction, which has a reported kinematic viscosity at $20{ }^{\circ} \mathrm{C}$ of 5.26 centistokes. ${ }^{30}$ (For comparison, the viscosity of pure water at $20{ }^{\circ} \mathrm{C}$ is 1.01 centistokes. ${ }^{30}$ ) The particles were then suspended in the viscous $\mathrm{pH}$ buffers, yielding a concentration of approximately $1 \times 10^{8}$ particles/mL, where they were magnetized and then sonicated for several minutes. Changes in $\mathrm{pH}$, measured with a $\mathrm{pH}$ meter, were within $0.1 \mathrm{pH}$ units after the addition of glycerol and the particles. However, we believe that any observed minor changes were related to loose barium ferrite particles that were not encapsulated in the silica matrix during synthesis. An alternative method that we have developed in parallel takes advantage of a new method ${ }^{22}$ that uses cobalt or iron coated with silver or gold, which avoids such chemical effects and, therefore, any changes in $\mathrm{pH}$. Finally, each solution was pipetted into two different homemade fluidic cells, with a typical chamber thickness of $\sim 100 \mu \mathrm{m}$, width of $\sim 6.5 \mathrm{~mm}$, and length of $\sim 22$ $\mathrm{mm}$.

Microscopy. All microscopic images were obtained with a Roper Coolsnap ES charge coupled device (CCD) camera (Roper Scientific Tucson, AZ) connected with an Olympus IMTII (Lake Success, NY) inverted epifluorescence microscope; see Figure 2. A Lambda DG-4 xenon lamp (Sutter Instruments, Novato, CA) and an Olympus blue filter cube were used for fluorescence studies. The objective lens used for bright-field images, fluorescent images, and fluorescence spectroscopy was an Edmund Optics $60 \times$ magnification lens with a numerical aperture of 0.85 . Spectroscopic data were obtained with an Acton Research Corp. spectrometer connected to a Hamamatsu 230 CCD. The camera frame rate for experiments performed under bright-field microscopy was $\sim 32$ frames/s, while fluorescence images were obtained in a single image acquisition mode. To observe a significant signal for the acquisition of fluorescence spectra, we suggest that a minimum of three $1-10$ $\mu$ m particles should be in the microscope's field of view, which, for our synthesis, was approximately equivalent to weight percent of solids of $0.1 \%$ of sensing particles to water.

Magnetic Particle Rotation. Particles were observed in the aforementioned $\sim 100-\mu \mathrm{m}$-thick fluidic cell near a glass - water interface, at a distance of a micrometer or less. Rotation was carried out with a digital function generator that was used to rotate a stepper motor connected to a $5 / 8$ in. diameter, diametrically magnetized Alnico magnet (Dexter Magnetics, Inc.). The magnet was placed in a vertical orientation $\sim 12 \mathrm{~cm}$ to the side of the sample so that the magnetic field was rotated in the focal plane with a magnitude of $\sim 6$ Oe and at speeds ranging from 0 to 2.5 rotations/s. The particle was observed to be 
(a) In-Plane Rotation of Asymmetric Particles

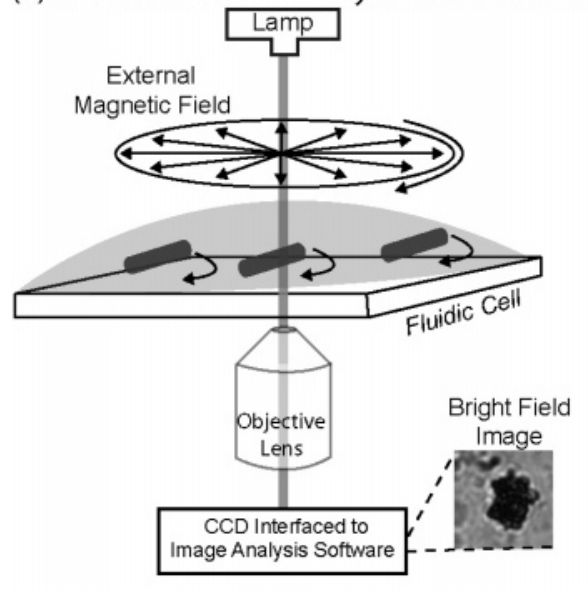

(b) Fluorescence Spectroscopy Setup

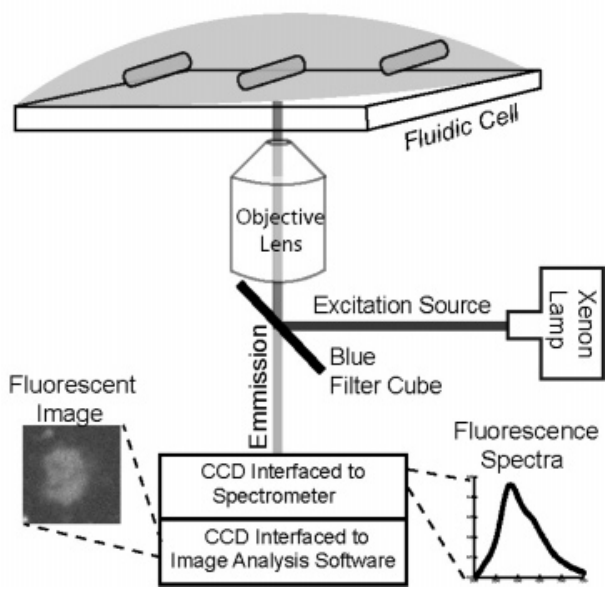

Figure 2. Experimental setup used for (a) the rotation of asymmetric particles, viewed using bright-field microscopy, and (b) fluorescent spectroscopy. The particles were contained in a $\sim 100-\mu \mathrm{m}$-thick fluidic cell (not shown). Image insets show a typical particle, $\sim 10 \mu \mathrm{m}$ in length, under brightfield and fluorescent microscopy.

actively rotated by this external rotation field, and since no translation was observed, any magnetic gradient forces produced by the external field were negligible. Also, no significant gradient is expected over the microdomain of one particle. Generally, the $\mathrm{pH}$-sensing magnetic particle aligned with the field along its easy axis of magnetization (along its length). The glass interface confined the motion to two dimensions, reducing tumbling and simplifying the analysis.

Analysis. Images were acquired and analyzed using Metamorph software (Universal Imaging Corp., Sunnyvale, CA) with bright-field microscopy. The Metamorph particle tracking function was used to track in time the vertical displacement of the corner of a magnetic particle. For each rotation the corner of the particle will be furthest away at half a rotation and return back to the origin after one full rotation; see "In-Plane Rotation" in Figure 1. In this way, the vertical displacement roughly expresses the sine of the angle and made it possible to monitor the orientation of the particle. Then, a fast Fourier transform was applied to the time series to obtain the average rotation rate. This type of image analysis was only possible because of the shape asymmetry of the observed particle. Shape asymmetries similar to that of the particle used to obtain the data discussed in the Results and Discussion section was typical in our batches of synthesized particles.

\section{Results and Discussion}

Fluorescence Characterization of Microparticles. To ensure that the $\mathrm{pH}$-sensing magnetic particle contained fluorescent dye, we first checked the fluorescent images of the particles; for example, see "Fluorescent Image" in Figure 2b. The shape of the particle imaged with fluorescence microscopy is similar to the shape of the same particle under bright-field microscopy (see "Bright Field Image" in Figure 2a), indicating that fluorescent dye was concentrated within the particle. The sizes of the particles used in these experiments were on the order of $1-10 \mu \mathrm{m}$. The large particle size and irregular shapes may have resulted from aggregation of the barium ferrite nanoparticles during synthesis. The size of the particles discussed in this paper excludes their use for most cellular studies. However, $5 \mu \mathrm{m}$ particles have been attached to human bronchial epithelial cells and used to study the microrheology ${ }^{33}$ of the cells. Further refinement of our synthesis process or use of a new fabrication technique should allow for the production of sub-micrometer

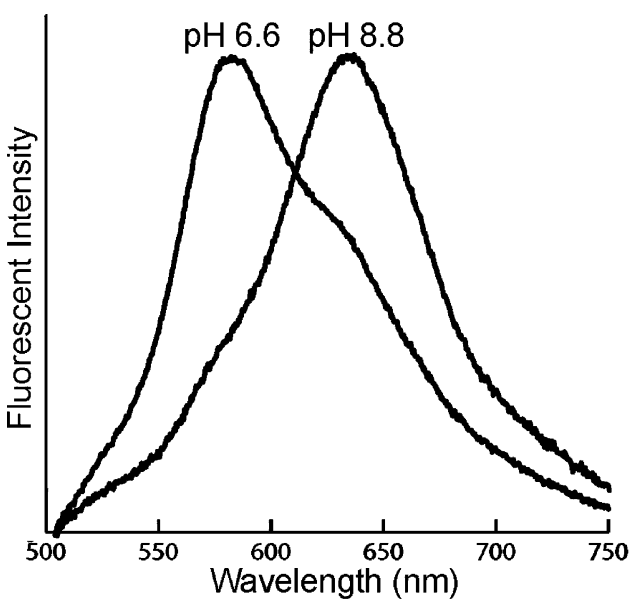

Figure 3. Average fluorescence emission spectra (normalized at isosbestic point) of $\mathrm{pH}$-sensing magnetic particles in a viscous $\mathrm{pH} 6.6$ buffer, peak location at $\sim 585 \mathrm{~nm}$, and a viscous $\mathrm{pH} 8.8$ buffer, peak location at $\sim 637 \mathrm{~nm}$.

PEBBLEs as small as $50 \mathrm{~nm}$ with uniform amounts of magnetic materials, ${ }^{22}$ although this would require a hemisphere metal capping, so that the change in particle orientation could be tracked.

After the fluorescent images of the particles were checked, the fluorescence emission spectra of the particles in $\mathrm{pH} 6.6$ and pH 8.8 glycerol buffer solutions were obtained. The resulting spectra, as shown in Figure 3, demonstrate that the particles were sensitive to a change of $\mathrm{pH}$ from 6.6 to 8.8. The location of the emission maximum changed from a short-wavelength acidic peak, at $\sim 585 \mathrm{~nm}$, to a longer-wavelength basic peak, at $\sim 637 \mathrm{~nm}$. This change in peak location is expected for SNARF$1{ }^{34}$ While there was a small amount of photobleaching, it did not occur at a rate that affected our measurements.

Sudden Breakdown of Linear Response: Critical PhaseSlipping. Having obtained pH-dependent spectra, bright-field microscopy was used to monitor the magnetic particle shown in Figure 4. Figure 4 shows a magnetic particle used in the critical phase-slipping experiments at different times and orientations before critical phase-slipping, top row, and after critical phase-slipping, bottom rows. At an external field rotation rate of $\pi / 2 \mathrm{rad} / \mathrm{s}(0.25$ rotations/s), the particle rotates in phase with the external magnetic field. At each time step, the particle progresses in the same clockwise direction-never rotating in a 


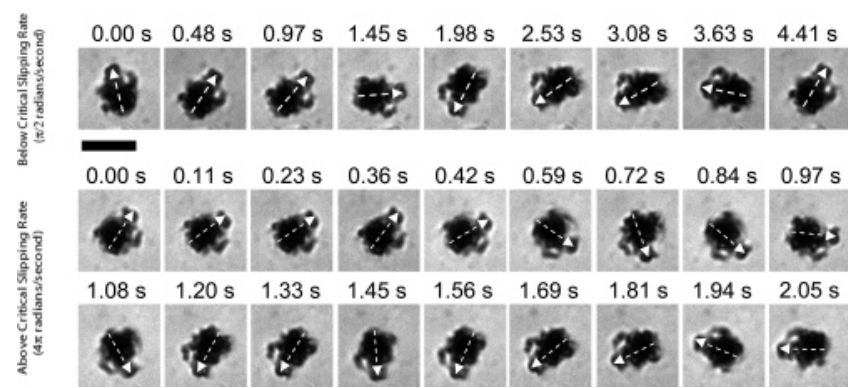

Figure 4. Sequence of bright-field microscopy images of a single $\mathrm{pH}$ sensing magnetic particle, where the black scale bar is $\sim 7 \mu \mathrm{m}$. Top row: The particle is driven at below the critical slipping rate, progressing in the clockwise direction. Bottom rows: The particle is driven at above the critical slipping rate. The particle begins in the clockwise direction, $t=0.00-0.23 \mathrm{~s}$ but then rotates in a direction direction, at $t=0.36,0.84,0.97,1.45$, and $2.05 \mathrm{~s}$.

direction opposite to the driving field-see top row of Figure 4. However, when the external field rotation rate is increased from $\pi / 2$ to $4 \pi \mathrm{rad} / \mathrm{s}$ (2.0 rotations/s, i.e., above the critical slipping rate), the particle generally rotates in the clockwise direction but occasionally rocks in the opposite direction. It is a result of the viscous drag becoming too high in comparison with the magnetic torque. This is the type of rotational behavior that causes the particle to have a lower average rotation rate than that of the external field.

Tracking a corner of the particle and plotting the vertical displacement that it travels from its origin allows for the measurement of the particle's average rotation rate. Figure 5 further reveals the critical phase-slipping behavior of the $\mathrm{pH}$ sensing magnetic particle shown in Figure 4. Before critical phase-slipping, Figure 5a, the vertical displacement appears to that is opposite of the external driving field, the counterclockwise

be sinusoidal in time, and the average rotation rate of the magnetic particle was found by taking a Fourier transform, Figure 5b (see Analysis in the Experimental Section). Above the critical slipping rate, Figure $5 \mathrm{c}$, the periodic behavior of the particle reveals a high-frequency oscillation, due to regular rocking being superimposed on the slower frequency oscillation that results from the gradual clockwise progression of the particle. The magnetic particle is being lapped by the external field, and to minimize torque, the particle momentarily "rocks" in a direction opposite to that of the external field. Thus, the particle is rocking once per rotation of the external field. These two oscillations are seen in the Fourier transform, Figure 5d, where the largest peak is the slower average rotation rate, $\sim 0.31$ $\mathrm{Hz}$, and the smaller peak is the faster rotation rate, $\sim 2.0 \mathrm{~Hz}$, of the external field, seen through the intermittent counterclockwise rotations of the particle.

The average rotation rate of the magnetic particle, at various external field rotation rates, was determined from data similar to that shown in Figure 5 and is summarized in Figure 6. The change of behavior due to phase-slipping can be seen as the external field rotation rate is increased. Initially, the relationship is linear, with a one-to-one correspondence, so that when the external rotation rate is doubled the average particle rotation rate is also doubled. However, at driving frequencies above the critical slipping rate, the rotational behavior is nonlinear, and the average rotation rate of the particle becomes slower for increasing external field rotation rates. The slower rotation rate is due to the frequent rocking (also referred to as "flip backs"9 or "swaying"10). Fitting eq 4 to the experimental data points, using a least-squares fit, gives a critical slipping rate of $\sim 6.8$ $\mathrm{rad} / \mathrm{s}$ (1.1 rotations/s).

The data in Figure 6 deviates slightly from the theoretical fit. Most notably, the particle's average speed goes down faster

\section{Fourier Transform (Average Rotation Rate)}
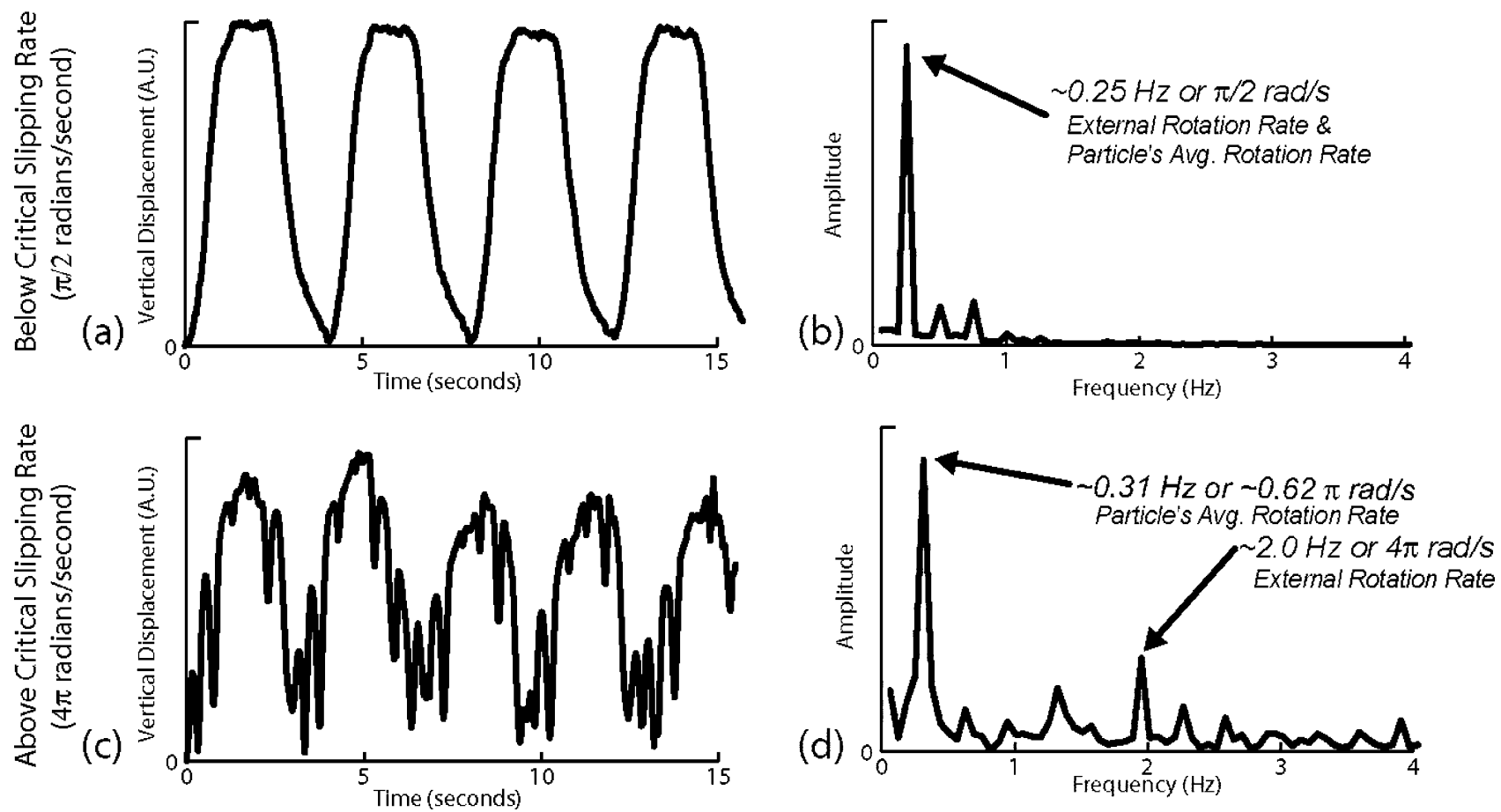

Figure 5. Rotational behavior seen by plotting the vertical displacement of a corner of a single aspherical pH-sensing magnetic particle shown in Figure 4, at two external rotation rates - (a) $\pi / 2 \mathrm{rad} / \mathrm{s}(0.25 \mathrm{rotations} / \mathrm{s})$ and (c) $4 \pi \mathrm{rad} / \mathrm{s}$ ( $2.0 \mathrm{rotations} / \mathrm{s})$. From the periodic data, a Fourier transform was taken to determine the average rotation rate, parts $b$ and $d$. Below the critical slipping rate, part $b$ shows only one main peak, which is a result of the particle being in phase with the external field (with a constant phase lag). At external rates above the critical slipping rate, part d reveals two main peaks-one that results from the slower net rotation of the particle and one that results from the faster counterclockwise rocking of the magnetic particle, which is at the same rate as the external field. 


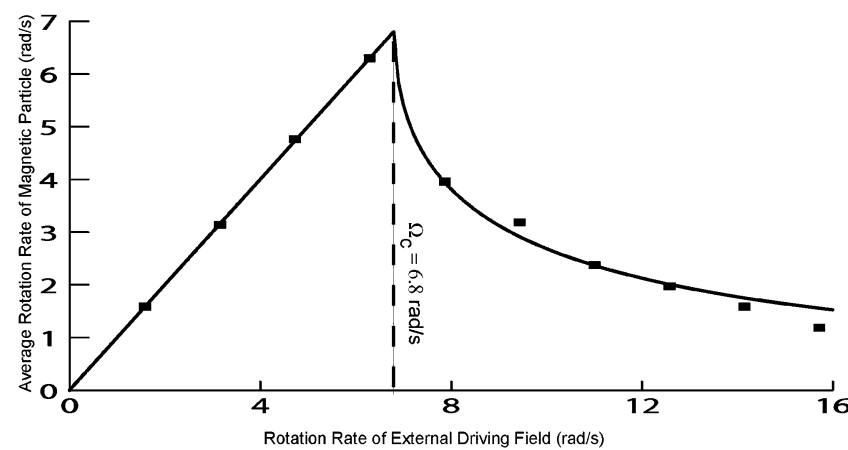

Figure 6. Critical phase-slipping behavior of a single asymmetric $\mathrm{pH}-$ sensing magnetic particle, where the squares are experimental data and the line is a least-squares fit. The critical slipping rate, calculated from the fit, is $\sim 6.8 \mathrm{rad} / \mathrm{s}$. The slope of the line, before the onset of critical phase-slipping, is simply equal to one.

than what would be expected with the theory of a particle in free solution. We expect some deviations from the theory, because the particle is near a glass interface. Despite the minor deviations from the theoretical fit in Figure 6, the critical slipping rate was fairly reproducible. While we were not able to reproduce the entire data set, shown in Figure 6, more than two times for a single particle-due to the particle eventually sticking to an interface or aggregating with other particles-we were able to estimate the critical slipping rate by slowly varying the rotation rate, from above and below the critical slipping rate, and watching for any rotations opposing the direction of the external field to occur. Through the use of this technique, the critical phase-slipping was reproducibly found to be in the range from $\sim 6.75$ to $\sim 7.2 \mathrm{rad} / \mathrm{s}$, which agrees with the critical slipping rate of $\sim 6.8 \mathrm{rad} / \mathrm{s}$ that was determined from fitting the data.

Being able to determine the critical slipping rate is useful because it is sensitive to physical properties such as viscosity, the particle's magnetic moment, external magnetic fields, and size; see Table 1. To obtain absolute measurements for any of these parameters, the other parameters must be known. Variation in shape and magnetic composition limits the accuracy of such measurements and requires single-particle calibration or ensemble measurements. This is a result of the particle-to-particle nonuniformities in size, shape, and magnetic content. However, if the same particle is monitored while parameters of the system are altered, then measurements with only a single particle can be quite useful. For example, in past experiments we succeeded in monitoring a change in the critical slipping rate, $\Omega_{\mathrm{c}}$, of a single MagMOON when the temperature in a highly viscous glycerol solution was changed. ${ }^{35}$ To obtain improved particleto-particle uniformity, further refinement of the synthesis technique or use of a new fabrication technique is needed. One promising fabrication method is to half-coat nonmagnetic PEBBLEs with a magnetic material, such as cobalt or iron. ${ }^{22}$ We have also observed critical phase-slipping with these probes and their uniformity was only limited by the starting uniformity of the PEBBLEs.

Further experimentation with critical phase-slipping and refinement of fabrication methods should allow for straightforward measurements of viscosity, temperature changes, singleparticle magnetic moment, rotating magnetic fields, or even volume changes due to a biological antigen attaching to the particle. This, in combination with the ability to measure chemical concentrations, will make possible a powerful new kind of micro- or nanosensor that could probe physical and chemical properties in biological environments.

\section{Conclusions}

We demonstrated a multiple-sensing microplatform by measuring a change in $\mathrm{pH}$, from 6.6 to 8.8 , and by measuring the critical slipping rate of a single magnetic particle. The critical slipping rate is sensitive to physical values such as the viscosity and the volume of the particle. The ability to measure $\mathrm{pH}$ changes in combination with the onset of phase-slipping; i.e., the sudden breakdown of the linear response to external field rotation indicates that this sensor can be used for probing in tandem physical and chemical properties of a microenvironment. The further development and miniaturization of sensing magnetic particles that can probe both physical and chemical properties may allow for applications in biologically interesting environments, such as inside a live embryo or the cytoplasm of cell.

Supporting Information Available: The authors acknowledge Thomas Horvath for help with the synthesis setup, Keith D. Bonin from Wake Forest University for helpful theoretical discussions, Rodney Agayan for helpful discussions regarding theory and the experimental setup, and the University of Michigan Electron Microbeam Analysis Laboratory. This work was funded by the National Science Foundation (Grant No. DMR 0455330) and a DARPA BioMagnetICs grant.

\section{References and Notes}

(1) Monson, E.; Brasuel, M.; Philbert, M. A.; Kopelman, R. In Biomedical Photonics Handbook; Vo-Dinh, T., Ed.; CRC Press: Boca Raton, FL, 2003.

(2) Clark, H. A.; Hoyer, M.; Philbert, M. A.; Kopelman, R. Anal. Chem. 1999, 71, 4831 .

(3) Clark, H.; Kopelman, R.; Tjalkens, R.; Philbert, M. A. Anal. Chem. 1999, 71, 4837 .

(4) Brasuel, M.; Kopelman, R.; Miller, T. J.; Tjalkens, R.; Philbert, M. A. Anal. Chem. 2001, 73, 2221.

(5) Xu, H.; Aylott, J. W.; Kopelman, R.; Miller, T. J.; Philbert, M. A. Anal. Chem. 2001, 73, 4124.

(6) Anker, J. N.; Behrend, C. J.; McNaughton, B. H.; Roberts, T. G.; Brasuel, M.; Philbert, M. A.; Kopelman, R. Mater. Res. Soc. Symp. Proc. 2004, 790, 4.4.1.

(7) Roberts, T. G.; Anker, J. N.; Kopelman, R. J. Magn. Magn. Mater. 2005, 293, 715.

(8) Crick, F. H. C.; Hughes, A. F. W. Exp. Cell Res. 1950, 1, 37.

(9) Shelton, A. W.; Bonin, K. D.; Walker, T. G. Phys. Rev. E 2005, 71, 036204

(10) Biswal, S. L.; Gast, A. P. Anal. Chem. 2004, 76, 6448.

(11) Korneva, G.; Ye, H.; Gogotsi, Y.; Halverson, D.; Friedman, G.; Bradley, J. C.; Kornev, K. G. Nano Lett. 2005, 5, 879.

(12) Moskun, A. C.; Jailaubekov, A. E.; Bradforth, S. E.; Tao, G.; Stratt R. M. Science 2006, 311, 1907.

(13) Helgesen, G.; Pieranski, P.; Skjeltorp, A. T. Phys. Rev. Lett. 1990, $64,1425$.

(14) Yamazaki, A.; Sendoh, M.; Ishiyama, K.; Hayase, T.; Arai, K. I. Sens. Actuators, A 2003, 105, 103.

(15) Behrend, C. J.; Anker, J. N.; McNaughton, B. H.; Kopelman, R. J. Magn. Magn. Mater. 2005, 293, 663.

(16) Anker, J. N.; Horvath, T.; Kopelman, R. Eur. Cells Mater. 2002, 3,34 .

(17) Anker, J. N.; Behrend, C. J.; Kopelman, R. J. Appl. Phys. 2003, 93, 6698 .

(18) Anker, J. N.; Kopelman, R. Appl. Phys. Lett. 2003, 82, 1102.

(19) Anker, J. N.; Behrend, C. J.; Kopelman, R. J. Magn. Magn. Mater. 2005, 293, 655 .

(20) Behrend, C. J.; Anker, J. N.; McNaughton, B. H.; Brasuel, M.; Philbert, M. A.; Kopelman, R. J. Phys. Chem. B 2004, 108, 10408.

(21) McNaughton, B. H.; Anker, J. N.; Kopelman, R. J. Magn. Magn. Mater. 2005, 293, 696.

(22) McNaughton, B. H.; Stoica, V.; Anker, J. N.; Clarke, R.; Kopelman, R. Fabrication of uniform half-shell magnetic nanoparticles and microspheres with applications as magnetically modulated optical nanoprobes; 2005. http:// arxiv.org/abs/cond-mat/0506418. 
(23) Jesorka, A.; Markstrom, M.; Karlsson, M.; Orwar, O. J. Phys. Chem. $B$ 2005, 109, 14759.

(24) Glogauer, M.; Ferrier, J.; McCulloch, C. A. G. Am. J. Physiol.: Cell Physiol. 1995, 269, C1093.

(25) Van Vliet, K. J.; Bao, G.; Suresh, S. Acta Mater. 2003, 51, 5881

(26) Bao, G.; Suresh, S. Nat. Mater. 2003, 2, 715.

(27) Valberg, P. A.; Butler, J. P. Biophys. J. 1987, 52, 537.

(28) Purcell, E. M. Am. J. Phys. 1977, 45, 3.

(29) Strogatz, S. H. Nonlinear Dynamics and Chaos; Westview Press: Cambridge, MA, 2000

(30) Shankar, P. N.; Kumar, M. Proc. R. Soc. London, Ser. A 1994, $444,573$.
(31) Stober, W.; Fink, A.; Bohn, E. J. Colloid Interface Sci. 1968, 26, 62.

(32) Nozawa, K.; Gailhanou, H.; Raison, L.; Panizza, P.; Ushiki, H.; Sellier, E.; Delville, J. P.; Delville, M. H. Langmuir 2005, 21, 1516.

(33) Puig-de-Morales, M.; Grabulosa, M.; Alcaraz, J.; Mullol, J.; Maksym, G. N.; Fredberg, J. J.; Navajas, D. J. Appl. Physiol. 2001, 91, 1152 .

(34) Haugland, R. P. The Handbook-A Guide to Fluorescent Probes and Labeling Technologies, 10th ed.; Invitrogen: Carlsbad, CA, 2005. http:// probes.invitrogen.com/handbook/.

(35) McNaughton, B. H. et al., to be published. 